\title{
Can ACEI/ARB prevent the cardiotoxicity caused by chemotherapy in early-stage breast cancer? - a meta-analysis of randomized controlled trials
}

\author{
Haoran Dong ${ }^{1,2 \#}$, Litong Yao ${ }^{1 \#}$, Mozhi Wang ${ }^{1,2}$, Mengshen Wang ${ }^{1,2}$, Xinyan Li $^{1,2}$, Xiangyu Sun ${ }^{1,2}$, \\ Xueting Yu ${ }^{1}$, Jingyi Guo ${ }^{1}$, Xiang $\mathrm{Li}^{1}$, Yingying $\mathrm{Xu}^{1}$ \\ ${ }^{1}$ Department of Breast Surgery, the First Affiliated Hospital of China Medical University, Shenyang, China; ${ }^{2}$ Department of Surgical Oncology and \\ General Surgery, Key Laboratory of Precision Diagnosis and Treatment of Gastrointestinal Tumors, Ministry of Education, The First Affiliated \\ Hospital of China Medical University, Shenyang, China \\ Contributions: (I) Conception and design: H Dong, L Yao, Y Xu; (II) Administrative support: Y Xu; (III) Provision of study materials or patients: H \\ Dong, L Yao; (IV) Collection and assembly of data: M Wang, M Wang, X Li, X Li; (V) Data analysis and interpretation: X Sun, X Yu, J Guo; (VI) \\ Manuscript writing: All authors; (VII) Final approval of manuscript: All authors. \\ \#These authors contributed equally to this work. \\ Correspondence to: Professor Yingying Xu. Department of Breast Surgery, the First Affiliated Hospital of China Medical University, 155 North \\ Nanjing Street, Shenyang 110001, China. Email: xuyingying@cmu.edu.cn.
}

Background: Administration of anthracycline-based chemotherapy with or without trastuzumab is recognized as standard care for breast cancer, but it is associated with a decline in left ventricular ejection fraction (LVEF). Angiotensin-converting enzyme inhibitors (ACEI)/angiotensin II receptor blockers (ARB) might decrease this cardiac dysfunction caused by the anti-cancer therapy. We sought to evaluate the prophylactic effects of the cardioprotective agents ACEI/ARB for early-stage breast cancer.

Methods: We systematically searched the electronic databases Cochrane, PubMed, and Embase for randomized controlled trials (RCTs) evaluating the effect of ACEI/ARB. This meta-analysis calculated weighted mean differences with $95 \%$ CI, for ejection fraction and pooled odds ratios (OR) with $95 \%$ CI, for cardiac events. Pooled analyses were used in a random-effect model. The primary endpoint was the change of LVEF in the ACEI/ARB group versus the control group from baseline through completion of the studies. Results: our meta-analysis includes 5 studies encompassing 702 early-stage breast cancer patients. There was statistically significant diversity in the magnitude of the change of mean LVEF in patients receiving ACEI/ARB compared with control groups, with a mean difference of $4.08 \%$ (95\% CI: $0.8 \%$ to $7.35 \%$, $\mathrm{P}=0.01)$. However, regarding patient outcomes, ACEI/ARB did not significantly reduce the risk of cardiac events (OR 0.91, 95\% CI: 0.62 to $1.34, \mathrm{P}=0.64$ ) or increase the incidence of hypotension events as compared with controls (OR 2.72, 95\% CI: 0.69 to $10.73, \mathrm{P}=0.15$ ).

Conclusions: Our study suggests that ACEI/ARB significantly attenuate the cardiac dysfunction caused by anthracycline-based chemotherapy and/or trastuzumab. Further studies are required to confirm the effectiveness of this cardioprotective agent.

Keywords: Breast cancer; chemotherapy; cardiotoxicity; angiotensin-converting enzyme inhibitors (ACEI); angiotensin II receptor blockers (ARB); cardioprotection; meta-analysis

Submitted Apr 26, 2020. Accepted for publication Sep 18, 2020.

doi: $10.21037 /$ tcr-20-1869

View this article at: http://dx.doi.org/10.21037/tcr-20-1869 


\section{Introduction}

Breast cancer is one of the most common malignancies, accounting for approximately $15 \%$ of all cancer deaths and $25 \%$ of overall cancer cases in females $(1,2)$. While the development of diagnostic technologies and novel therapeutic methods have significantly improved survival for breast cancer patients in the past decades (3), systemic chemotherapy is still considered as the standard treatment for breast cancer (4). Unfortunately, the improved survival has been accompanied with cardiotoxic effects from the chemotherapeutic regimens, leading to elevated mortality (5). A previous study of patients treated for breast cancer confirmed that cardiovascular disease was the leading cause of death in the study population, closely followed by breast cancer (6). The incidence rate of heart failure in elderly breast cancer patients receiving anthracyclinebased chemotherapy and/or trastuzumab therapy was over twice that of patients who did not receive adjuvant therapy. Furthermore, the rate of heart failure was highest in patients treated with a combination of anthracycline and trastuzumab. Strategies for preventing this cardiovascular impairment have been deeply studied in recent years.

Cardiovascular injuries are defined as a greater than $10 \%$ drop in left ventricular ejection fraction (LVEF) from baseline, with the value sometimes reaching below $53 \%$ normal LVEF (7). Anthracycline is a primary chemotherapeutic agents with dose-dependent activity. Heart failure morbidity increases $5 \%$ when a cumulative dose of $400 \mathrm{mg} / \mathrm{m}^{2}$ anthracycline is administered, and this rises to $16 \%$ with a dose of $500 \mathrm{mg} / \mathrm{m}^{2}$ and $48 \%$ with a dose of $700 \mathrm{mg} / \mathrm{m}^{2}$ (8). Trastuzumab, a humanized monoclonal antibody against HER-2 commonly used in early-stage breast cancer adjuvant treatment, is also reported to result in an unexpectedly high incidence of adverse cardiovascular events (9). Therefore, cardioprotective strategies are warranted to prevent or alleviate cardiotoxic events for breast cancer patients. Angiotensin-converting enzyme inhibitors (ACEI) and angiotensin II-receptor blockers (ARB) both provide notable benefits for decreasing left ventricular dysfunction specifically caused by anthracycline therapy (10-12). ACEI have shown inhibitory effects on chemotherapy-induced cardiomyopathy in both animal models and adult patients with early cardiotoxicity, demonstrating that ACEI can reduce mortality in patients with anthracycline-induced cardiomyopathy $(13,14)$. Multiple completed and ongoing clinical trials have evaluated the efficacy of ACEI or ARB in preventing cardiac dysfunction in breast cancer. However, conflicting results have arisen. The objective of this meta-analysis is to estimate the influence of ACEI/ARBs on clinical cardiotoxicity caused by anthracycline-based chemotherapy with/without trastuzumab, with a focus on early-stage breast cancer. We present the following article in accordance with the PRISMA reporting checklist (available at http://dx.doi. org/10.21037/tcr-20-1869).

\section{Methods}

\section{Search strategy}

Systematic searches of the Embase, PubMed, and Cochrane library databases were performed using the following search terms: "breast cancer", "cardiotoxicity", "chemotherapy", "ACEI", and "ARB" for studies published through November 2019. Reference lists of obtained articles were checked to retrieve relevant studies. Data reported as abstracts only and unpublished data were excluded from this analysis. The search only included articles published in English.

\section{Selection criteria}

Two reviewers independently screened and selected the studies using the following inclusion criteria: randomized controlled trials (RCTs) involving early-stage breast cancer patients undergoing anthracycline-based chemotherapy with or without trastuzumab treatments; two arms including ACEI/ARB and placebo with an end point measurement of LVEF; and a minimal follow-up duration of 6 months from the initial therapy. LVEF was measured with echocardiography or cardiac magnetic resonance imaging (MRI). Cardiac events and hypotension events were defined according to the decisions made by the original articles. Although some trials included beta blockers, these arms were not analyzed in this study. In studies describing patients with different cancer types, only patients with breast cancer were included in this meta-analysis.

\section{Data extraction and quality assessment}

Two authors extracted data independently, referring to the Preferred Reporting Items for Systematic Reviews and Meta-Analyses (PRISMA) statement. Any discrepancies between the two reviewers were resolved by a third investigator. The following information was extracted from 


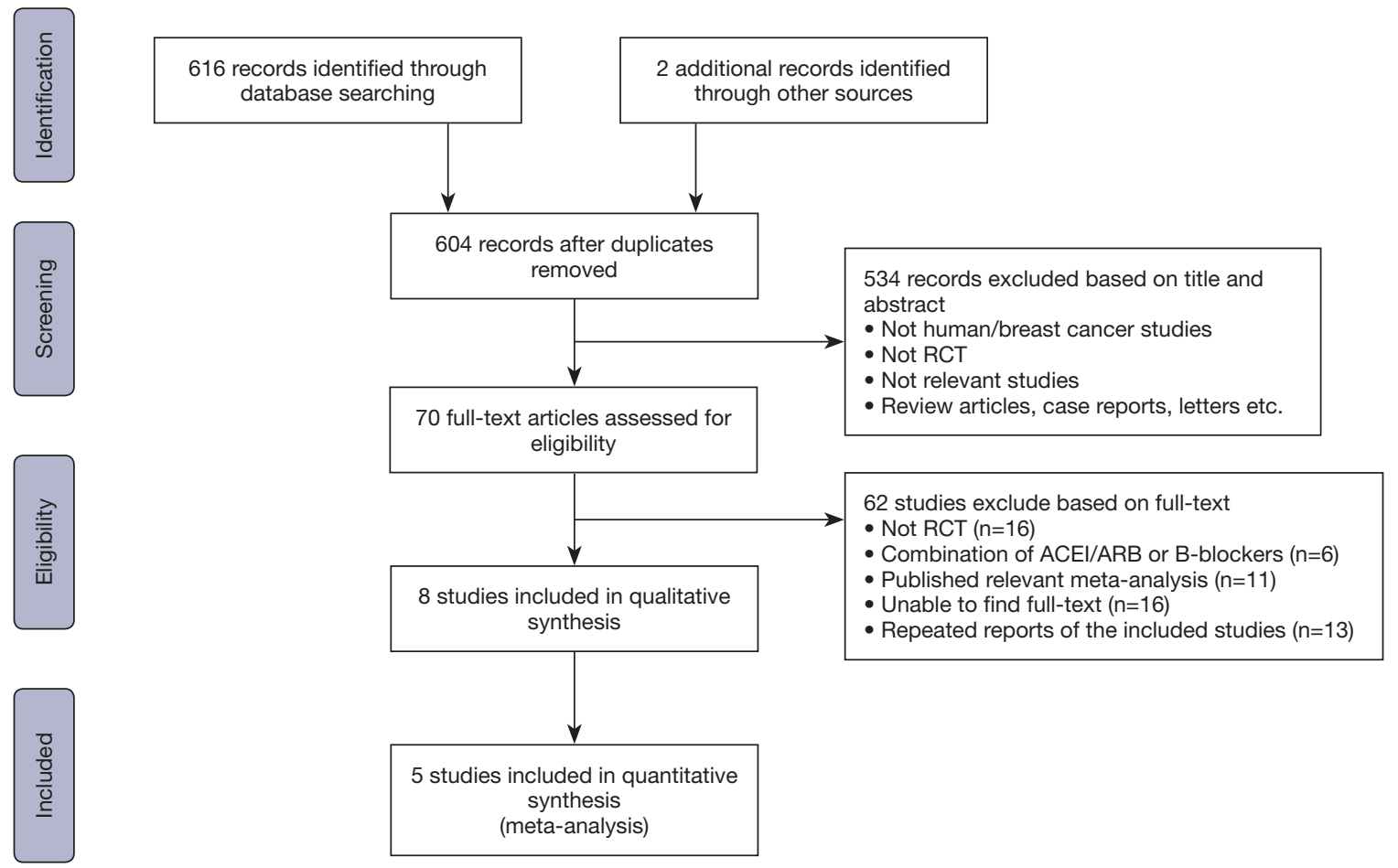

Figure 1 Flow diagram of literature search.

the included studies: study name, authors, publication year, study characteristics, number of participants enrolled, follow-up duration, and measurement of LVEF. The primary author was contacted to acquire additional information if there was insufficient information in the articles. The primary endpoint of this meta-analysis was the change of mean LVEF within the period from the baseline to study termination in the ACEI/ARB group compared with the control group. The secondary endpoint included baseline LVEF and the incidence of cardiac events and hypotension events caused by anti-cancer therapy. The risk of bias in the RCTs was assessed using the Cochrane Collaboration tool, which considers seven domains including adequacy of sequence generation, allocation concealment, blinding of participants and personnel, blinding of outcome assessment, incomplete outcome data, selective outcome reporting, and other bias. Discrepancies were resolved by discussion among authors.

\section{Statistical analysis}

The continuous variable for ejection fraction was analyzed using a weighted mean difference with a $95 \%$ CI, whereas dichotomous variables for cardiac events and hypotension events were analyzed using odds ratio (OR) and a 95\% CI. Study heterogeneity was estimated using the Cochran $\mathrm{Q}$ test and the $\mathrm{I}^{2}$ statistic. An $\mathrm{I}^{2}$ index of less than $25 \%$ was assigned as low heterogeneity, $25-50 \%$ as moderate heterogeneity, and more than $50 \%$ as high heterogeneity. The DerSimonian and Laird random effects model was applied to combine the potential heterogeneity to yield a more considerable result. All values were two-sided and $\mathrm{P}$-values of less than 0.05 were considered statically significant. A funnel plot was used to evaluate publication bias of the primary outcome. Review Manager (RevMan) version 5.3 software was used for the meta-analysis.

\section{Results}

The search flow for identifying relevant studies is outlined in Figure 1. A total of 618 studies were initially identified through the systematic search. We excluded 14 duplicates and screened 604 articles based on titles and abstracts, of which 70 full-text articles were evaluated for eligibility. Sixty-two further articles were excluded as 16 were not RCTs, 6 administered a combination of ACEI/ARB and $\beta$-blocker therapy, 11 were published relevant metaanalyses, we were unable to find full-text for 16 , and 13 
Table 1 Study design and patient characteristics.

\begin{tabular}{|c|c|c|c|c|c|c|c|c|c|}
\hline Study & Year & Country & $\begin{array}{l}\text { Type of } \\
\text { study }\end{array}$ & Chemotherapy & Arm & $\begin{array}{l}\text { Sample } \\
\text { size }\end{array}$ & $\begin{array}{l}\text { Follow-up } \\
\text { Duration } \\
\text { Months }\end{array}$ & Mean age & $\begin{array}{l}\text { Measurement of } \\
\text { LVEF }\end{array}$ \\
\hline Pituskin et al. & 2017 & Canada & $\mathrm{RCT}$ & $\begin{array}{l}\text { Anthracycline } \\
\text { and/or Trastuzumab }\end{array}$ & Placebo & 30 & 12 & $51 \pm 7$ & Cardiac MRI \\
\hline Boekhout et al. & 2016 & Netherlands & RCT & $\begin{array}{l}\text { Anthracycline } \\
\text { and/or Trastuzumab }\end{array}$ & Candesartan & 103 & 21 & 50 & Echocardiography \\
\hline \multirow[t]{2}{*}{ Janbabai et al. } & \multirow[t]{2}{*}{2017} & \multirow[t]{2}{*}{ Iran } & \multirow[t]{2}{*}{$\mathrm{RCT}$} & \multirow[t]{2}{*}{ Anthracycline } & Enalapril & 29 & 6 & 47.8 & \multirow[t]{2}{*}{ Echocardiography } \\
\hline & & & & & Placebo & 30 & & 48.8 & \\
\hline \multirow[t]{2}{*}{ Guglin et al. } & \multirow[t]{2}{*}{2019} & \multirow[t]{2}{*}{ USA } & \multirow[t]{2}{*}{$\mathrm{RCT}$} & \multirow{2}{*}{$\begin{array}{l}\text { Trastuzumab } \\
\text { and/or Anthracycline }\end{array}$} & Lisinopril & 158 & 24 & $50.58 \pm 10.91$ & \multirow[t]{2}{*}{ Echocardiography } \\
\hline & & & & & Placebo & 154 & & $51.11 \pm 10.32$ & \\
\hline
\end{tabular}

were repeated reports of included studies. Thus 8 of the 604 articles were identified as eligible. After careful review, we found that statistics for 3 studies were not available because the outcomes were assessed across different cancer types. We contacted the primary authors about assessment of breast cancer alone, and only one provided detailed information. Ultimately, 5 articles encompassing 702 breast cancer patients receiving chemotherapy were included for our meta-analysis.

Study design and patient characteristics are presented in Table 1. The follow-up duration varied from 6 months to 24 months. The chemotherapeutics which might result in cardiotoxicity for all included studies were anthracyclinebased chemotherapy regimens with/without trastuzumab. For the cardioprotective medications, ACEI and ARB agents such as candesartan, enalapril, perindopril and lisinopril were applied. For the measurement of LVEF, three studies used echocardiography and two used cardiac MRI. All RCTs included in this meta-analysis were classified as high quality, as shown by the bias summary (Figure 2).

All five included RCTs examined the potential cardioprotective effects ACEI/ARB had on the change in mean LVEF following anti-cancer therapy, with outcomes shown in Figure 3. Three hundred and fifty-five patients were designated into intervention groups receiving ACEI/ ARB treatment and 347 were designated into control groups which received placebo treatment. There was significant heterogeneity between studies ( $\mathrm{P}$ for Cochrane's $Q$ test $<0.00001, \mathrm{I}^{2}=93 \%$ ) and a stochastic effects model was used for pooled analysis. There was a statically significant difference in the magnitude of the change of mean LVEF from baseline in patients receiving ACEI/ARB compared with controls, with a mean difference of $4.08 \%$ (95\% CI: $0.8 \%$ to $7.35 \%, \mathrm{P}=0.01$ ). The baseline mean LVEF are shown in Figure 4. The results show no significant differences in LVEF status at baseline settings across the included studies.

Safety outcomes regarding incidence of cardiac events in patients undergoing chemotherapy showed that ACEI/ ARB therapy was not related to a significantly lower risk of cardiotoxicity compared to controls (Figure 5, OR $0.91,95 \%$ CI: 0.62 to $1.34, \mathrm{P}=0.64$ ) with no significant heterogeneity ( $\mathrm{P}$ for Cochrane's $\mathrm{Q}$ test $=0.1, \mathrm{I}^{2}=56 \%$ ) in a random-effect model. Three studies including 581 breast cancer patients reported the incidence of adverse hypotension events during the study, with 14 of 294 patients in the ACEI/ARB group and 4 of 287 in the control group being diagnosed with hypotension. ACEI/ARB treatment did not significantly improve the risk of hypotension events (Figure 6, OR 2.72, 95\% CI: 0.69 to $10.73, \mathrm{P}=0.15$ ) with a low heterogeneity ( $\mathrm{P}$ for Cochrane's $\mathrm{Q}$ test $=0.31, \mathrm{I}^{2}=16 \%$ ), but we did notice an obvious trend.

\section{Discussion}

Breast cancer is a significant public health problem worldwide, with incidence and mortality rates as high as $30 \%$ and $15 \%$, respectively (15). Due to improved systemic 

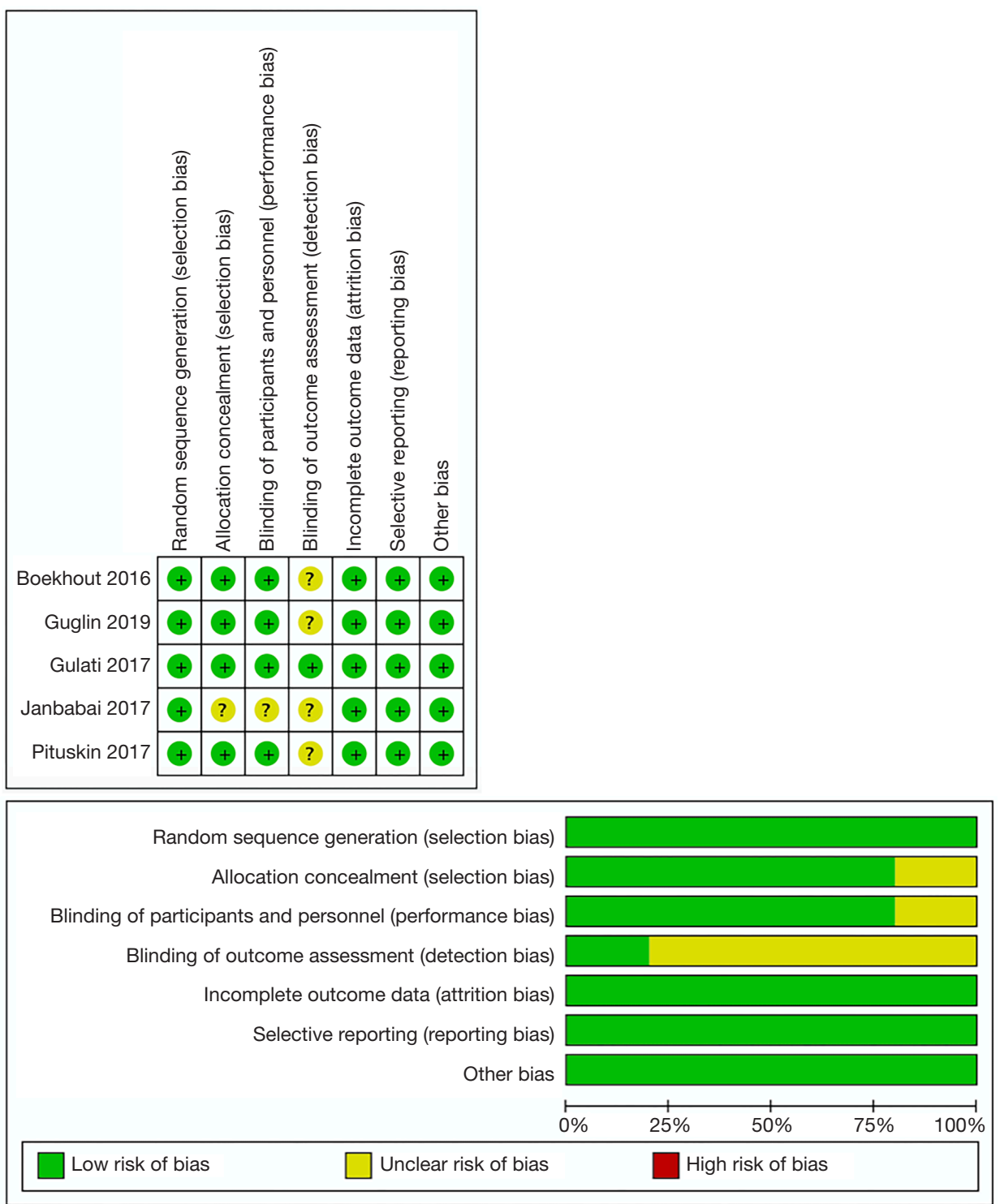

Figure 2 Risk of bias summary: review of authors' judgements about each risk of bias item for each included study.

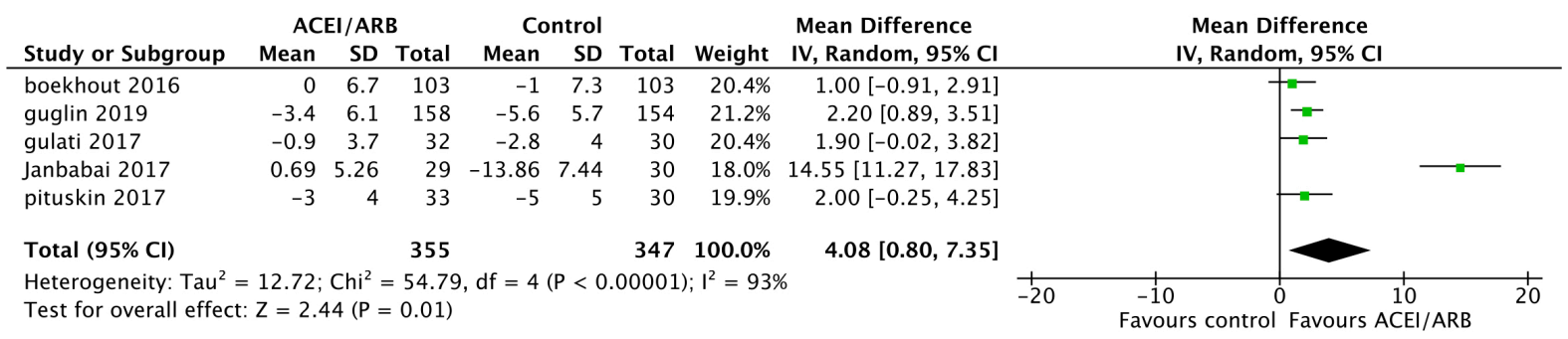

Figure 3 Forest plot for the mean change in LVEF with ACEI/ARB versus control. LVEF, left ventricular ejection fraction; ACEI, angiotensin-converting enzyme inhibitors; ARB, angiotensin II-receptor blockers. 


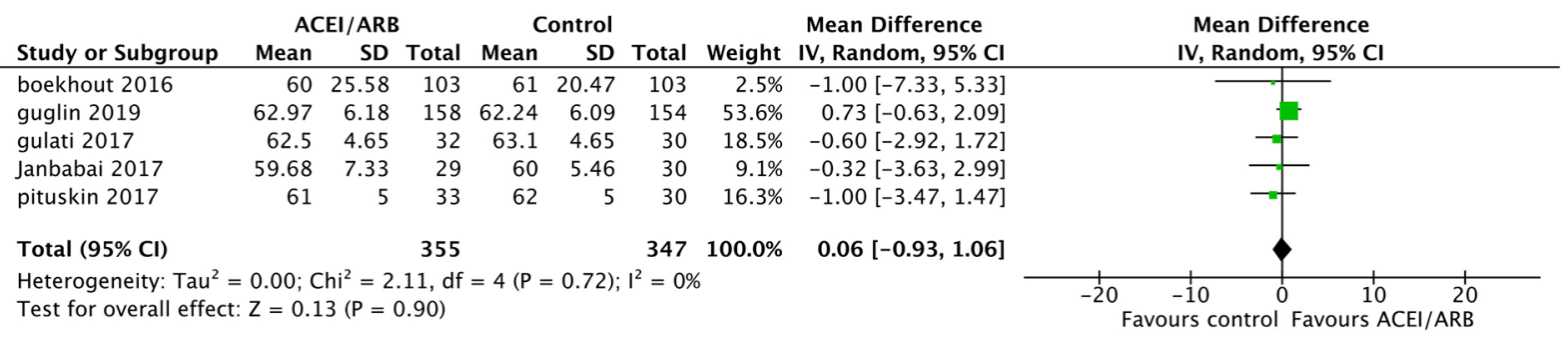

Figure 4 Forest plot for the baseline LVEF with ACEI/ARB versus control. LVEF, left ventricular ejection fraction; ACEI, angiotensinconverting enzyme inhibitors; ARB, angiotensin II-receptor blockers.

\begin{tabular}{|c|c|c|c|c|c|c|c|c|}
\hline Study or Subgroup & $\begin{array}{l}\text { ACEI/A } \\
\text { Events }\end{array}$ & $\begin{array}{l}\text { ARB } \\
\text { Total }\end{array}$ & $\begin{array}{l}\text { Contr } \\
\text { Events }\end{array}$ & $\begin{array}{l}\text { ol } \\
\text { Total }\end{array}$ & Weight & $\begin{array}{l}\text { Odds Ratio } \\
\text { M-H, Fixed, } 95 \% \mathrm{Cl}\end{array}$ & $\begin{array}{c}\text { Odds Ratio } \\
\text { M-H, Fixed, } 95 \% \mathrm{Cl}\end{array}$ & \\
\hline boekhout 2016 & 20 & 103 & 16 & 103 & $23.5 \%$ & $1.31[0.64,2.70]$ & & \\
\hline guglin 2019 & 45 & 158 & 46 & 154 & $60.8 \%$ & $0.93[0.57,1.52]$ & & \\
\hline gulati 2017 & 0 & 32 & 0 & 30 & & Not estimable & & \\
\hline Janbabai 2017 & 0 & 29 & 0 & 30 & & Not estimable & & \\
\hline pituskin 2017 & 3 & 33 & 9 & 30 & $15.6 \%$ & $0.23[0.06,0.97]$ & & \\
\hline Total $(95 \% \mathrm{Cl})$ & & 355 & & 347 & $100.0 \%$ & $0.91[0.62,1.34]$ & & \\
\hline Total events & 68 & & 71 & & & & & \\
\hline \multicolumn{7}{|c|}{$\begin{array}{l}\text { Heterogeneity: } \mathrm{Chi}^{2}=4.51, \mathrm{df}=2(\mathrm{P}=0.10) ; \mathrm{I}^{2}=56 \% \\
\text { Test for overall effect: } Z=0.46(P=0.64)\end{array}$} & $\begin{array}{lcc}0.01 & 0.1 & 1 \\
& \text { Favours ACEI/ARB } & \text { Favours C }\end{array}$ & $\begin{array}{l}10 \\
\text { control }\end{array}$ \\
\hline
\end{tabular}

Figure 5 Forest plot for the risk of cardiac events with ACEI/ARB versus control. ACEI, angiotensin-converting enzyme inhibitors; ARB, angiotensin II-receptor blockers.

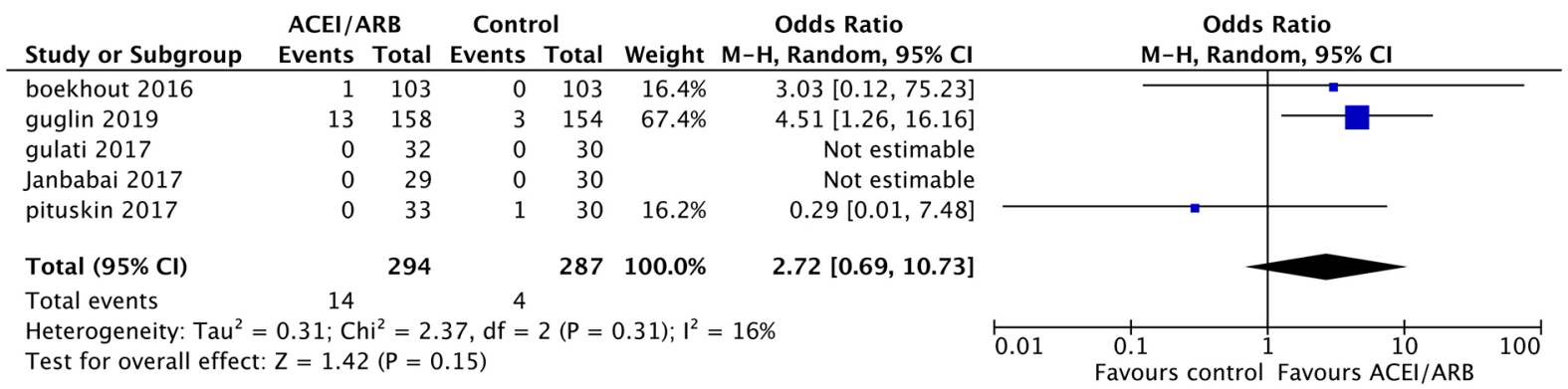

Figure 6 Forest plot for the risk of hypotension with ACEI/ARB versus control. ACEI, angiotensin-converting enzyme inhibitors; ARB, angiotensin II-receptor blockers.

treatment agents and radiotherapy, the survival rate for breast cancer patients has significantly improved in recent years. Unfortunately, the cardiotoxicity induced by standard chemotherapy strategies administering anthracycline with or without trastuzumab raises the risk for cariological harm in patients receiving treatments for cancer. This increased attention to cardiotoxicity induced by anti-cancer treatments propagated the new field of cardio-oncology. ACEI/ARB were confirmed as one of the most common prophylactic therapies and revealed a mitigative effect on cardiotoxicity in several studies. Previous meta-analyses have evaluated the potential cardioprotective effects of ACEI/ARB in various types of cancer $(16,17)$. Our meta-analysis focuses on breast cancer, which significantly threatens the survival of female 
patients. Our analysis included 5 studies involving 702 patients and we confirmed that prophylactic ACEI/ARB has a critical cardioprotective effect, with a significant reduction of change in LVEF (4.08\%) in patients receiving ACEI/ ARB treatment group versus the control group. However, we found no significant relationship between prophylactic ACEI/ARB therapy and decreased risk of chemotherapyrelated cardiac events. Moreover, the ACEI/ARB group showed a 2.72-fold decreased risk of hypotension events compared to the control group, but this difference was not statistically significant. Thus, ACEI/ARB may be beneficial for breast cancer patients who suffer from cardiac dysfunction caused by anthracycline-based chemotherapy and/or trastuzumab.

The PRADA study (18), a $2 \times 2$ factorial randomized placebo-controlled trial, was designed to examine whether ARB (candesartan) or beta-blocker (metoprolol) concomitant therapy relieves the cardiotoxicity associated with anthracycline-containing chemotherapy regimens and/ or trastuzumab in adjuvant settings and radiation. LVEF was found to decline $2.6 \%$ in the placebo group and $0.8 \%$ in the candesartan group. In the MANTICORE 101Breast trial (19), patients with HER2-positive breast cancer were randomized to undergo perindopril $(n=33)$ or placebo $(\mathrm{n}=30)$ treatment, and the trastuzumab-related decline of mean LVEF was significantly attenuated in the ACEI group $(-3 \pm 4 \%)$ as compared to the placebo $(-5 \pm 5 \%)$ group in cardiotoxicity analysis. Janbabai et al. discovered that LVEF remained the same at baseline and 6-month postchemotherapy in the enalapril group while it was reduced in the control group, consistent with the above studies (14). Furthermore, in a recent clinical trial (NCT01009918) presented in June 11, 2019 (20), 468 patients were randomly appointed into lisinopril, carvedilol, or placebo groups. The study suggested that cardiotoxicity-free survival was improved in both the lisinopril (hazard ratio HR 0.53) and carvedilol (HR 0.49) groups compared to the placebo group. Patients receiving anthracyclines suffered a higher rate of cardiac events when administered placebo (47\%) compared to lisinopril (37\%) or carvedilol (31\%). We included data from the ACEI/ARB arm of this study into our metaanalysis. However, Boekhout et al. did not identify a cardioprotective effect for candesartan used concomitantly with common chemotherapy for breast cancer patients. Candesartan did not change the NTproBNP or hsTnT values and showed no significant effect on LVEF. Therefore, we conducted this meta-analysis to corroborate these clinical articles to more fully investigate whether prophylactic ACEI/ARB can alleviate LV dysfunction in breast cancer patients receiving anthracycline-based chemotherapy regimens with or without trastuzumab. Further studies are required to identify the effects of ACEI/ $\mathrm{ARB}$ as cardioprotective agents.

Multiple underlying mechanisms have been proposed for anthracycline-based chemotherapy-mediated cardiotoxicity. Currently, reactive oxygen species (ROS) production contributing to oxidative stress represents the most extensively studied explanation. A previous study revealed that ROS formation was induced by a crucial mediator of anthracycline-induced cardiotoxicitythe isozyme topoisomerase $2 \beta$ (TOP $2 \beta$ ). The TOP $2 \beta$ doxorubicin complex can further facilitate changes in genes related to oxidative phosphorylation and mitochondrial biogenesis in cardiomyocytes. Investigators determined that the cardiotoxicity of anthracyclines might be caused by the combination of anthracycline products with DNA forming complexes and ultimately impairing cardiomyocytes (21). Several studies have established mouse models of doxorubicin-induced cardiac dysfunction to evaluate the potential prophylactic mechanisms of ACEI and ARB. ACEI/ARB were found to participate in cardiomyocyte proliferation, differentiation, and apoptosis, and they further helped preserve myocardial morphology and function via stimulation of ERK and the PI3K/AKT/mTOR pathway (22). While the mechanism of trastuzumab-induced cardiotoxicity is still debated, it is known to cause reversible dysfunction not associated with cell loss, tending to result in type II adverse drug reactions instead of type I adverse drug reactions which can lead to irreversible damage. HER2 receptors are generally expressed in cardiomyocytes, and the presence of trastuzumab activates the HER2 signaling pathway, generating moderate oxidative stress, oxidative status imbalance of cardiomyocytes, and ultimately reversible cardiac dysfunction (23). Further investigations analyzing mechanisms for the cardioprotective role of ACEI/ARB in trastuzumab treatment settings are warranted.

The clinical benefits of ACEI have been distinctly illustrated for a series of cardiovascular conditions including acute myocardial infarction, asymptomatic left ventricular dysfunction, chronic heart failure hypertension, and in patients with a raised risk of cardiovascular events. Although the mechanisms by which ACEI improve clinical outcomes in patients with systolic dysfunction are not thoroughly understood, induction of more beneficial hemodynamic 
conditions and the countervailing effects of RAS activation likely impact this process. Previous studies have confirmed that ACEI can attenuate afterload and systolic ventricular wall stress, improve cardiac output, decrease the progrowth effects of angiotensin II on myocytes, increase ventricular geometry, and reduce aldosterone-induced cardiac fibrosis and apoptosis of cardiac cells. Additionally, cardiac angiotensin-converting enzyme (ACE) activity has been indicated to be increased after chemotherapy compared to control groups. Treatment with lisinopril after chemotherapy significantly inhibited cardiac ACE activity and improved cardiac remodeling, mortality, and cardiac dysfunction in vivo. Lisinopril has been further demonstrated to suppress collagen accumulation and prevent fibrosis in the cardiac interstitium. These results show that the benefits of ACEI in anthracycline-treated breast cancer patients are related to inhibition of cardiac ACE, whose activation is crucial in the development of this cardiomyopathy (24). ACEI have been further studied in recent years. The ICOS-ONE Study was the first multicenter randomized trial that compared two schemes to prevent anthracycline-induced cardiotoxicity with enalapril. One strategy was designed to prevent the troponin rise caused by anthracyclines by treating all patients with cardioprotective therapy combined with chemotherapy, while the other strategy only gave enalapril to select patients who had increasing troponin during or after chemotherapy. The study showed that incidence of troponin climbed during anthracycline-treatment and the major endpoint was identical in both groups independent of enalapril treatment (25). There were also studies proofed that ARBs has a protectective effect on rats treated with anthracyclines; it indicated that pretreatment with the ARBs reduced cardiac tissue damage (26) .

However, the disadvantages of ACEI are also obvious. ACEI may reduce heart rate, lower blood pressure, and contribute to fatigue. As a result, these changes make patients receiving anti-cancer treatment less resistant to ACEI. These patients are already fatigued by the cancer itself, the corresponding treatment, anemia, and intravascular volume depletion resulting from inadequate oral intake, vomiting, and diarrhea. Thus, this impaired tolerance has encouraged clinicians to consider alternative cardio-protective medications such as betablockers and statins. Several studies have determined the cardioprotective effects for these two agents, but efficacy and safety comparisons are rare and give conflicting results. Furthermore, LVEF monitoring is limited by variabilities between observers and time, and the decline may start in a later period after substantial cardiac injury (27). The relationship between LVEF and outcomes in HF patients is inconsonant. The present standard for measuring cardiac function is to monitor cardiotoxicity exclusively when functional injuries have already emerged, but this is not used for early cardioprotective therapies. Moreover, evaluation of cardio-specific biomarkers could be an efficient diagnostic tool for detecting early stage cardiotoxicity then evaluating and monitoring its progression. This method is minimally invasive and cheaper compared to echocardiography. Importantly, nuclear technology can easily be replicated without irradiating the patients. Also, for some biomarkers such as troponins and NP (Natriuretic peptides) (28), results can be consistently interpreted regardless of the expertise of the operator, avoiding variation between observers. Further studies are needed to provide specific guideline for widespread clinical use of such biomarkers.

One major limitation of our study is the significant heterogeneity between the results, largely due to the limited amount of studies meeting the inclusion criteria. Some RCTs included patients with different cancer types, with data specific to the breast cancer patient group unavailable from the pooled results. Furthermore, the follow-up duration for each therapy ranged from 6 to 24 months, different chemotherapy protocols and drug dosages were used without standardization, enrolled sample sizes varied from 60 to 312 , diverse preventative medications were used, and patient characteristics such as cardiovascular risk factors, predisposition to disease, potential LV dysfunction, co-morbidities, and compliance all added to the high heterogeneity. Another crucial shortcoming is the lack of uniform modality for LVEF measurement, and data from 2D-echocardiogram and cardiac MRI present inevitable variability, as these are subjective interpretations between observers. The endpoint of LVEF is also defined differently across studies. Finally, other parameters such as BNP and GLS were not uniformly assessed across the studies. However, the SAFE trial (NCT2236806) is evaluating the influence of bisoprolol, ramipril, or both agents combined compared to placebo in 480 patients (29). This currently ongoing prospective clinical trial may help reduce some of these limitations. The clinical trial by Lee et al. involving 485 patients also shows a significantly reduced incidence of anthracycline-induced subclinical cardiotoxicity and lower $\mathrm{LV}$ dysfunction in the ARB group compared to the control group (30). Our results are consistent with these studies. 


\section{Conclusions}

ACEI/ARB have crucial effects on decreasing the amount of LVEF decline caused by anthracycline-based chemotherapy and/or trastuzumab, making it potentially indispensable for breast cancer patients who suffer from cardiac dysfunction. Further studies are required to determine if it significantly reduces the incidence of cardiotoxicity events and improves survival for early-stage breast cancer patients after anticancer therapy.

\section{Acknowledgments}

This work was supported by National Natural Science Foundation of China (81773083), Scientific and Technological Innovation Leading Talent Project of Liaoning Province (XLYC1802108) and Support Project for Young and Technological Innovation Talents of Shenyang (RC190393).

\section{Footnote}

Reporting Checklist: The authors have completed the PRISMA reporting checklist. Available at http://dx.doi. org/10.21037/tcr-20-1869

Conflicts of Interest: All authors have completed the ICMJE uniform disclosure form (available at http://dx.doi. org/10.21037/tcr-20-1869). The authors have no conflicts of interest to declare.

Ethical Statement: The authors are accountable for all aspects of the work in ensuring that questions related to the accuracy or integrity of any part of the work are appropriately investigated and resolved.

Open Access Statement: This is an Open Access article distributed in accordance with the Creative Commons Attribution-NonCommercial-NoDerivs 4.0 International License (CC BY-NC-ND 4.0), which permits the noncommercial replication and distribution of the article with the strict proviso that no changes or edits are made and the original work is properly cited (including links to both the formal publication through the relevant DOI and the license). See: https://creativecommons.org/licenses/by-nc-nd/4.0/.

\section{References}

1. Carbine NE, Lostumbo L, Wallace J, et al. Risk- reducing mastectomy for the prevention of primary breast cancer. The Cochrane database of systematic reviews 2018;4:CD002748.

2. Torre LA, Bray F, Siegel RL, et al. Global cancer statistics, 2012. CA Cancer J Clin 2015;65:87-108.

3. Allemani C, Matsuda T, Di Carlo V, et al. Global surveillance of trends in cancer survival 2000-14 (CONCORD-3): analysis of individual records for 37513025 patients diagnosed with one of 18 cancers from 322 population-based registries in 71 countries. Lancet 2018;391:1023-75.

4. Early Breast Cancer Trialists' Collaborative Group (EBCTCG). Effects of chemotherapy and hormonal therapy for early breast cancer on recurrence and 15year survival: an overview of the randomised trials. Lancet 2005;365:1687-717.

5. Curigliano G, Cardinale D, Dent S, et al. Cardiotoxicity of anticancer treatments: Epidemiology, detection, and management. CA Cancer J Clin 2016;66:309-25.

6. Patnaik JL, Byers T, DiGuiseppi C, et al. Cardiovascular disease competes with breast cancer as the leading cause of death for older females diagnosed with breast cancer: a retrospective cohort study. Breast Cancer Res 2011;13:R64.

7. Kirkham AA, Paterson DI, Prado CM, et al. Rationale and design of the Caloric Restriction and Exercise protection from Anthracycline Toxic Effects (CREATE) study: a 3-arm parallel group phase II randomized controlled trial in early breast cancer. BMC Cancer 2018;18:864.

8. Mavrogeni SI, Sfendouraki E, Markousis-Mavrogenis G, et al. Cardio-oncology, the myth of Sisyphus, and cardiovascular disease in breast cancer survivors. Heart Fail Rev 2019;24:977-87.

9. Nemeth BT, Varga ZV, Wu WJ, et al. Trastuzumab cardiotoxicity: from clinical trials to experimental studies. Br J Pharmacol 2017;174:3727-48.

10. Yeh ET, Bickford CL. Cardiovascular complications of cancer therapy: incidence, pathogenesis, diagnosis, and management. J Am Coll Cardiol 2009;53:2231-47.

11. Boekhout AH, Gietema JA, Milojkovic Kerklaan B, et al. Angiotensin II-Receptor Inhibition With Candesartan to Prevent Trastuzumab-Related Cardiotoxic Effects in Patients With Early Breast Cancer: A Randomized Clinical Trial. JAMA Oncol 2016;2:1030-7.

12. Huang G, Zhai J, Huang $X$, et al. Predictive value of soluble ST-2 for changes of cardiac function and structure in breast cancer patients receiving chemotherapy. Medicine 2018;97:e12447. 
13. Cardinale D, Colombo A, Lamantia G, et al. Anthracycline-induced cardiomyopathy: clinical relevance and response to pharmacologic therapy. J Am Coll Cardiol 2010;55:213-20.

14. Janbabai G, Nabati M, Faghihinia M, et al. Effect of Enalapril on Preventing Anthracycline-Induced Cardiomyopathy. Cardiovasc Toxicol 2017;17:130-9.

15. Siegel RL, Miller KD, Jemal A. Cancer statistics, 2019. CA Cancer J Clin 2019;69:7-34.

16. Gujral DM, Lloyd G, Bhattacharyya S. Effect of prophylactic betablocker or ACE inhibitor on cardiac dysfunction \& heart failure during anthracycline chemotherapy \pm trastuzumab. Breast 2018;37:64-71.

17. Totzeck M, Mincu RI, Heusch G, et al. Heart failure from cancer therapy: can we prevent it? ESC Heart Fail 2019;6:856-62.

18. Gulati G, Heck SL, Ree AH, et al. Prevention of cardiac dysfunction during adjuvant breast cancer therapy (PRADA): a 2 × 2 factorial, randomized, placebocontrolled, double-blind clinical trial of candesartan and metoprolol. Eur Heart J 2016;37:1671-80.

19. Pituskin E, Mackey JR, Koshman S, et al. Multidisciplinary Approach to Novel Therapies in Cardio-Oncology Research (MANTICORE 101-Breast): A Randomized Trial for the Prevention of Trastuzumab-Associated Cardiotoxicity. J Clin Oncol 2017;35:870-7.

20. Guglin M, Krischer J, Tamura R, et al. Randomized Trial of Lisinopril Versus Carvedilol to Prevent Trastuzumab Cardiotoxicity in Patients With Breast Cancer. J Am Coll Cardiol 2019;73:2859-68.

21. Renu K, Abilash VG, Tirupathi Pichiah PB, et al. Molecular mechanism of doxorubicin-induced cardiomyopathy - An update. Eur J Pharmacol 2018;818:241-53.

22. Hullin R, Metrich M, Sarre A, et al. Diverging effects of enalapril or eplerenone in primary prevention against

Cite this article as: Dong $\mathrm{H}$, Yao L, Wang M, Wang M, Li X, Sun X, Yu X, Guo J, Li X, Xu Y. Can ACEI/ARB prevent the cardiotoxicity caused by chemotherapy in early-stage breast cancer? - a meta-analysis of randomized controlled trials. Transl Cancer Res 2020;9(11):7034-7043. doi: 10.21037/tcr-20-1869 doxorubicin-induced cardiotoxicity. Cardiovasc Res 2018;114:272-81.

23. Zagar TM, Cardinale DM, Marks LB. Breast cancer therapy-associated cardiovascular disease. Nat Rev Clin Oncol 2016;13:172-84.

24. Cardinale D, Colombo A, Sandri MT, et al. Prevention of high-dose chemotherapy-induced cardiotoxicity in highrisk patients by angiotensin-converting enzyme inhibition. Circulation 2006;114:2474-81.

25. Cardinale D, Ciceri F, Latini R, et al. Anthracyclineinduced cardiotoxicity: A multicenter randomised trial comparing two strategies for guiding prevention with enalapril: The International CardioOncology Society-one trial. Eur J Cancer 2018;94:126-37.

26. Di Lisi D, Leggio G, Vitale G, et al. Chemotherapy cardiotoxicity: cardioprotective drugs and early identification of cardiac dysfunction. J Cardiovasc Med (Hagerstown) 2016;17:270-5.

27. Zhang KW, Finkelman BS, Gulati G, et al. Abnormalities in 3-Dimensional Left Ventricular Mechanics With Anthracycline Chemotherapy Are Associated With Systolic and Diastolic Dysfunction. JACC Cardiovasc Imaging 2018;11:1059-68.

28. Cardinale D, Sandri MT. Role of biomarkers in chemotherapy-induced cardiotoxicity. Progress in cardiovascular diseases 2010;53:121-9.

29. Meattini I, Curigliano G, Terziani F, et al. SAFE trial: an ongoing randomized clinical study to assess the role of cardiotoxicity prevention in breast cancer patients treated with anthracyclines with or without trastuzumab. Med Oncol 2017;34:75.

30. Lee MH, Chung WB, Song BJ, et al. The role of candesartan or carvedilol for prevention of anthracycline induced subclinical cardiotoxicity in breast cancer patients. Eur J Heart Fail 2019;21:328. 